\title{
SERVICE ORIENTED MODEL DRIVEN ARCHITECTURE FOR DYNAMIC WORKFLOW CHANGES
}

\author{
Leo Pudhota, Elizabeth Chang \\ PudhotaL@cbs.curtin.edu.au \\ School of Information Systems, Curtin University of Technology PO Box U1987, Perth WA 6845, Australia
}

Keywords: $\quad$ Services oriented, Collaborative Workflow, Logistics model, Isolation, Service integration

\begin{abstract}
Collaborative workflow management systems in logistic companies require strong information systems and computer support. These IT integration requirements have expanded considerably with the advent of $e$ business; utilizing web services for B2B (Business to Business) and P2P (Partner to Partner) e-commerce. This paper proposes service oriented model driven architecture for dynamic workflow changes and strategy for implementation of these changes by isolation of services and business processes where by existing workflow systems can easily incorporate and integrate the changes following a step by step process replacement synchronization in workflow. This paper will also describe conceptual framework for prototype implementation resulting in dynamic collaborative workflow management.
\end{abstract}

\section{INTRODUCTION}

In this paper we discuss the design of workflow management system for dynamic business processes of large logistic consortia. Often we see that the business processes are composed of several parts, a structured operational part and an unstructured operational part, or they could be composed of semi-structured parts with some given and some unknown details. Unpredictable situations may occur as a result of changes in decisions made by the management. The inability to deal with various changes greatly limits the applicability of workflow systems in real industrial and commercial operations. This situation raises problems in workflow design and workflow systems development. We propose workflow prototype implementation through service oriented architecture and system isolation for making changes to the existing workflow.

\section{DYNAMISM IN COLLABORATIVE WORKFLOW}

The advent of the web is to bind organizations together, for carrying out sales over great distances and at any time has created new modes for marketing and enabled partnerships, previously inconceivable within a wide array of businesses, as well as other human activities[1]. A consequence of this connectivity and information richness is that one is faced with an increasingly dynamic business environment and marketplace. This environment requires major form of collaborative workflow. A workflow is a sequence of activities that produces a result of observable value. A collaborative workflow is to focus on working together towards common goals. They can be small group of companies, project-oriented research teams, to widely dispersed industries with common interests. Effective 
use of collaborative workflow is now considered a vital element in the success of enterprises of all kinds. Workflow can be represented by sequence diagram, a collaboration diagram, Petri net or an activity diagram [5]. This IT support has expanded with the advent of ecommerce. However, with this advancement of B2B (Business to Business) and P2P (Partner to Partner) ecommerce [6], there has been an increasing tendency to set up consortia that represent several players in a given field. Such consortia consist of companies or organizations in a given field that get together and produce a single site or what appears to be single site in order to increase traffic through the site compared to other competitor's sites and/or extend beyond their region of operation, but a mere enumeration of all workers, activities and artefacts does not quite constitute a process. We need a way to describe meaningful sequences of activities that produce some valuable result, and to show interactions between processes.

Collaborative workflow management systems of a business sector like logistics consortium with multiusers and very dynamic environments will have: workflow specification, workflow execution, workflow evolution, workflow auditing, transaction management, workflow recovery, workflow interaction (for cooperative work), and others.

The specification of a workflow consists of three items:

Process: This includes the workflow tasks and how they are related. There should be enough flexibility to allow various forms of task interrelationships:

1. Tasks operating in series or in parallel

2. Tasks receiving input from or providing (possibly distinct) input to multiple other tasks

3. Tasks choosing to receive input from among many possible tasks that provide it

4. Tasks receiving input directly or indirectly from themselves (feedback)

5. Tasks being abstracted and grouped into higher level tasks

Data: This includes the input and output data of the workflow tasks.

Invocation: This includes the mechanism (rule) that triggers the execution of each task. There are two main choices: explicit invocation, in which a human initiates the task, and implicit invocation, in which the task begins immediately upon creation of its input as long as any specified conditions are satisfied.

Changes in a workflow may be an every-day routine in a working environment. Such changes are of three types:
Modification: new workflow has same objective but different logic and replaces old one.

Versioning: as before but new workflow does not replace old one, but co-exists with it.

Extension: new workflow has different objective and therefore additional logic and replaces old one.

In addition, some environments require dynamic rather than static workflow evolution, i.e., changing one part of the workflow while another part is running.

\section{WEB SERVICES FOR COLLABORATIVE LOGISTIC WORKFLOW}

An example of Warehouse and Logistics Consortium is to provide space for customers who want to store their goods in warehouse and shift their goods from origin location to destination location, detailed logistic services for its customer to move their goods from one place to another place. They have many types of logistic orders and each order has its own flow of work. Some of these orders are: Import: means that customer wants Consortium to pick up goods from one place and bring it to Consortium's warehouse. These goods are coming from outside Australia and the goods have to be declared/checked at Customs Office before entering Australia. So it is usually picked from either seaport or airport.

Export: means that customer wants consortium to deliver customer's goods from Consortium's warehouse to either seaports or airports outside Australia. These goods also need to be declared/checked before leaving Australia.

Local Delivery: means that customer wants consortium to deliver customer's good from one place to another place within Australia. All these orders involve other types of works, Devanning and Upvanning. Devanning is the process where goods are moved from trucks and put to warehouse. However, the process is not this simple. Some goods-checking procedures have to be done to put goods in right warehouse (e.g. frozen fish has to be put in cold-warehouse) or to find out if there is any discrepancies between the number of goods in the order and the actual number of goods that are coming to warehouse. Upvanning is simply the opposite of Devanning. Figure1 shows the basic services, which consortium provides to its customer. 


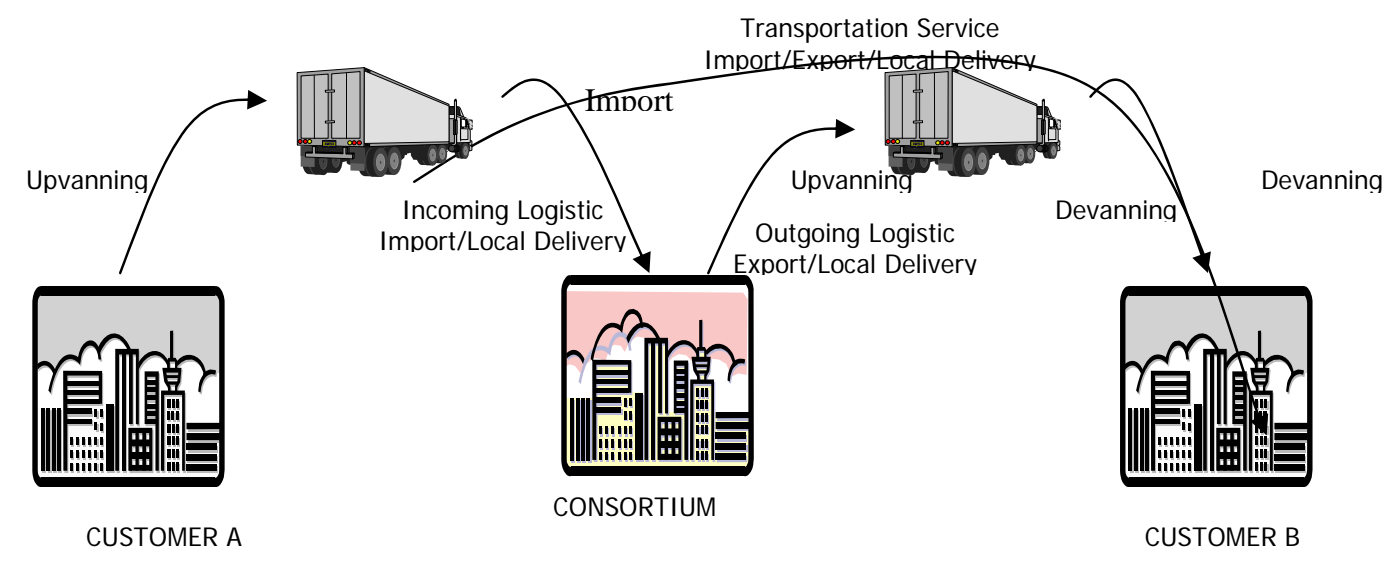

Figure 1: Example of collaborative logistic workflow.

On the other hand Logistic Management, in its widest definition, is concerned with the strategy and management of the movement and storage of materials and products from suppliers, through the firm's distribution system to retail outlets and customer. The scope of logistic management for the physical movement of goods starts with the sources of supply and ends at the point of consumption [2, 4]. In today's business environment, good logistics management often determines the success of a business. Retailers are well aware of how excess inventory, frequent stock-outs, poor item turnover, and excessive markdowns can cut into profits. Logistic management attempts to achieve a balance between holding minimum stock while providing the best services possible to the customer.

A Consortium consists of many departments; generally there are six operational divisions: Management Department, Warehouse Department, Logistic Department, Accounts Department, Customer Service Department and Transport Department. Each department has its own responsibility. However they are connected to each other. Warehouse Department now already has its own system, so does Accounts Department. The complexity of works become bigger and bigger when the customer's order increase. It is hard to know the progress of the orders and warehouse check. It is also difficult to schedule the trucks, manpower, etc. Consortium likes to change its internal work (flow of works among department) and its external work (flow of works with its customers and other collaborative organizations). Consortium would like to integrate various departments, and also with other logistic network companies in its consortium. Consortium also wants its customers to be able to book warehouse service, logistic service, place orders and view the status of orders, etc on the internet. This is more like e-commerce way. Figure 2 shows a typical eservices for collaborative workflow [3]. Here sellers are logistics providers, buyers are customers and web services brokers are web services integrators, Universal Description, Discovery and Integration providers Consortium is interlinked through internet and services are provided by web services. The basic premise behind Web Services is that a piece of code is made available to remote machines, using specific protocols, over the Internet. The Service part of Web Services relates to the idea of providing access to functionality without having to download or install the code, and the Web part refers to the means through which the functionality is accessed [19].The three component standards of Web Services are the Simple Object Access Protocol (SOAP), Universal Description, Discovery and Integration (UDDI), and Web Services Description Language (WSDL.). 


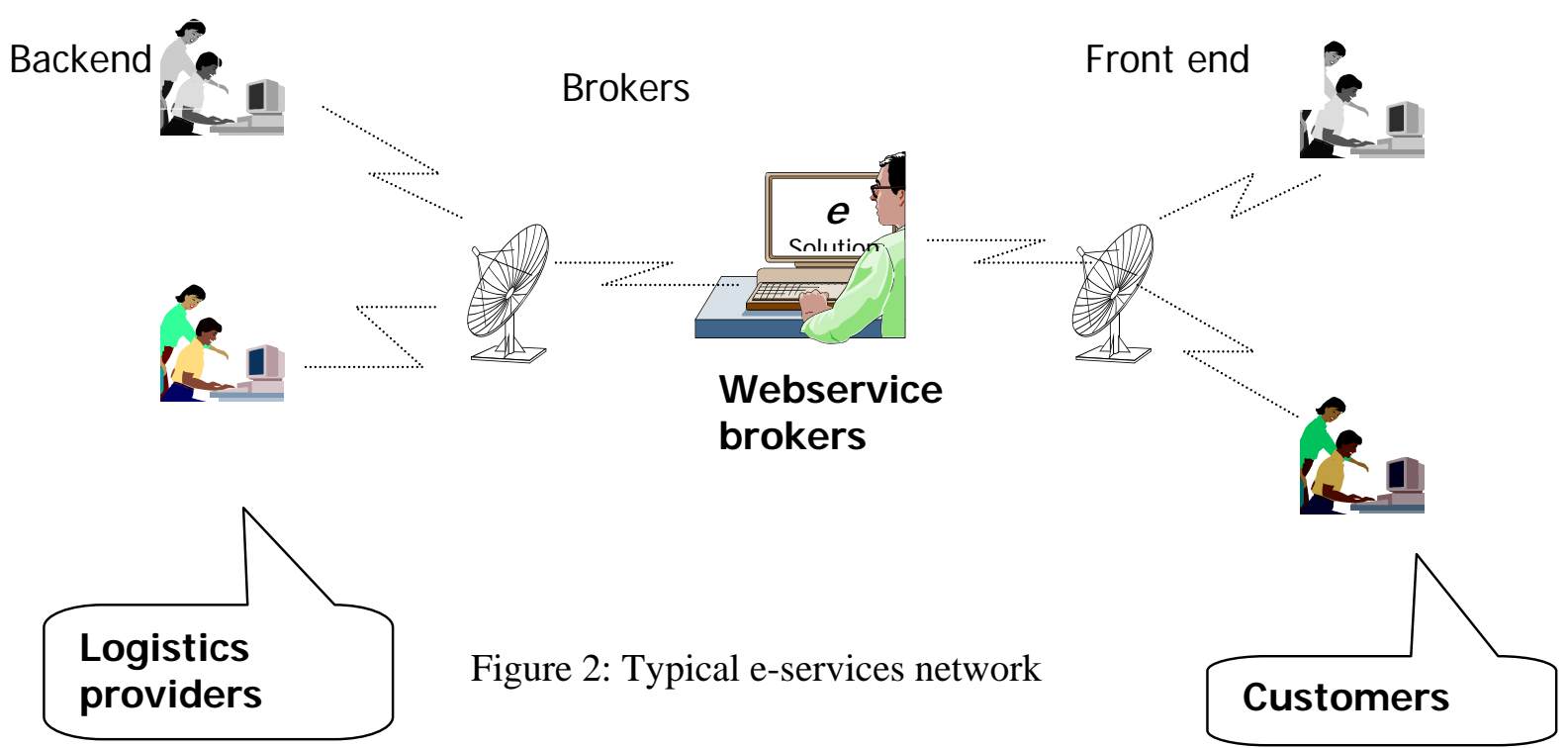

UDDI describes a registry in which Web Services can be accessed for reasons like, speed of the process, the need for trust, and the quality of the data in the UDDI registry. Consortium provides Web Service to members in the collaboration, once a business relationship is established between the company and the consortium and once this stage is reached business would continue as normal [19].

\section{ISSUES OF DYNAMISM COLLABORATIVE WORKFLOW}

Activities and artefacts do not quite constitute a process. We need a way to describe meaningful sequences of activities that produce some valuable result, and to show interactions between processes. Changes in collaborative workflow have to be incorporated into the integrated enterprise system. [8, 9, 10, 11].

In this paper we are concentrating on,

1. Design and Implementation of integrating solution for adaptation of changes in the new workflow into an already existing workflow.

2. Synchronization of new workflow to existing workflow.

Other issues like Management of data scattered over multiple origin systems/legacy systems, for example, a company will have consolidate data in one logical view with a unified architecture, thereby enabling datasource independence. Because application data continues to live and change in the origin systems, the new software layer must be able to retrieve origin data on the fly and also propagate changes back to the origin systems [17]. Provide support for transactions/interaction across multiple back-end systems.

These issues will help in having a uniform data processing environment for the whole enterprise, which would lead to changes and improvements in customer services, control of receivables and increase efficiency in communication, sales, marketing as well as minimization of warehouse stocks, streamlining inventory and logistics flows.

Provide control to Consortium management to monitor the collaborative enterprise's condition, its stock, order and its general financial condition on a routine basis, This is indispensable to the management processes and enhances decision-making and changes which need to be taken on the short term and long term bases for the consortium to compete in the global market. 


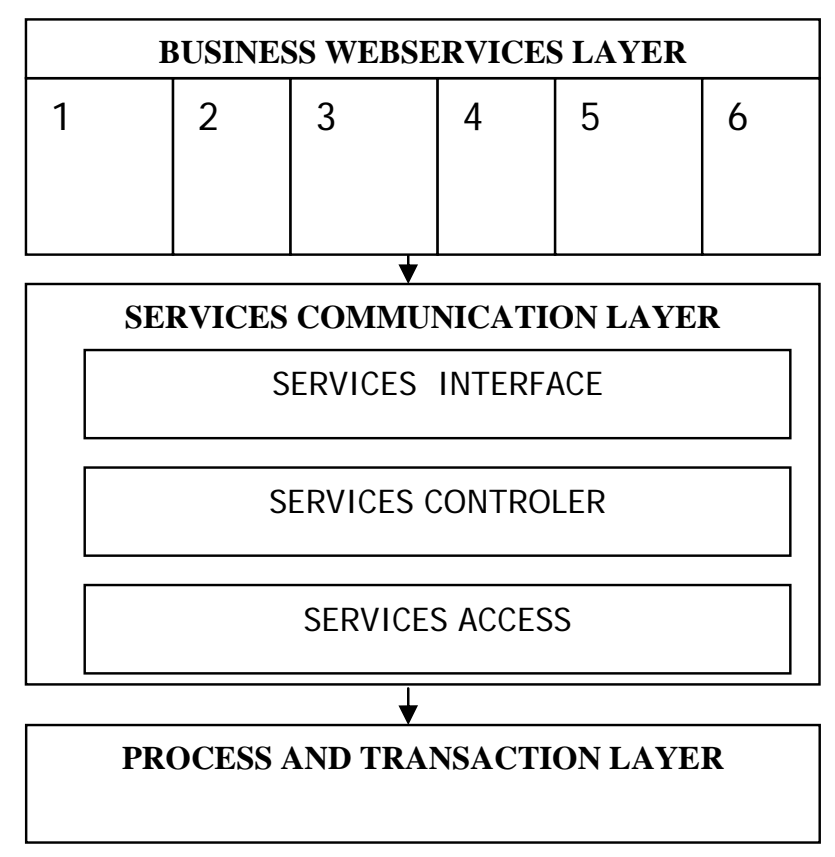

Figure 3. Enterprise model Frame work

\section{SERVICE ORIENTED FRAMEWORK TO SUPPORT BACKEND COLLABORATIVE WORKFLOW}

In this paper we present a service oriented framework for collaborative logistic companies. The framework is divided in 3 sections 1. Business web services layer. 2. Services communication layer and 3. Process and transaction layer. In Business web services layer browsers interact with HTTP servers in their normal way taking advantage of any technologies that enhance this browser-to-web server link. For example secure socket layer communication protocols in Netscape and Microsoft browser/server products browsers communicate with HTTP servers, which communicate with the Application Server. The Business web services layer generates web application at run time, Services communication layer provides application's user interface, state management and provides an environment to use and create reusable components [7]. Enterprise model framework shown in figure 3, balances across one or more application server processes (also called instances) running on one or more machines. Once running, Enterprise service framework instances do not go away between user requests; they maintain themselves, their session's state for users, and their database connections. They are efficient, fast, and by definition redundant. It's the job of the HTTP server adaptor to communicate with a given HTTP server and forward requests to one or more application "instances" - an instance is a separate copy of a given application process. Enterprise services framework serving a few users may have only one instance. A large application may have tens or hundreds of instances running on one or more machines. If an application has more than one instance, the Services controller is essentially acting as load balancing agent. If an instance fails, it only affects that particular instance - all other instances and/or the site's web server is unaffected. The controller will forward requests over the network as easily as it will forward requests to applications running on the same box as the HTTP server. In fact, from a load sharing perspective, it is ideal for the HTTP server and Application servers to reside on separate boxes. Since applications are server based, database access happens behind the firewall. Browsers need never make direct connections to a database server. Services access controls database connections so that they are highly secure (only accessible via actual application API), and conserved (that is, you never have more than one connection per 


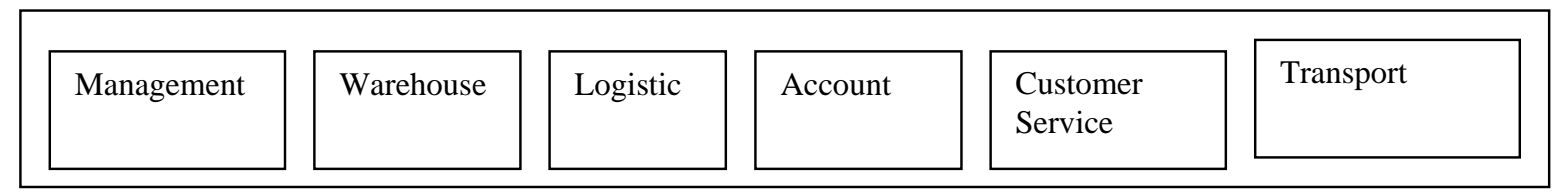

Figure 4: Business service layer for system

service unless this is specifically something the developers insist regardless of the number of users it supports). Process and Transaction layer works on underlying java foundation containing fundamental data structure, implementations and utilities used.

\section{CONCEPTUAL MODEL OF BUSINESS WEBSERVICES LAYER}

Referring to figure 4, we see each department has its own responsibility; however they are connected to each other. In a collaborative context, communication may have to be coordinated not only with in the organizations but also across organizations. Therefore a consortium may require synchronized coordination of activities of inter and intra organizational departments. This Conceptual Model provides an architectural separation of business functionality from technology implementation. This separation allows designers to use business rules defined in a UML model to drive two distinct steps in implementing such systems.

Step1. Create platform independent models in UML. The first model is a generic domain model, used to build a common understanding and vocabulary among warehouse Logistics domain experts. Step2. The domain model is then mapped into a representing warehouse logistic business. Each of the models includes a detailed set of UML Class Diagrams, Use Cases and associated Activity Diagrams describing the system [12].

\section{SERVICE COMMUNICATION LAYER}

This logical architecture of the web services frameworks (please refer to figure 3) is a programming building blocks of the largest granularity. Web services Frameworks is responsible in providing application's user interface and state management. Since applications are server based, database access happens behind the firewall. Browsers need never make direct connections to a database server. Services access controls database connections so that they are highly secure (only accessible via actual application API), and conserved (that is, you never have more than one connection per instance regardless of the number of users supported - unless this is specifically something the developers desire). Designers can use business rules as defined in previous section to define in UML model. Using this business model, we can create one or more subsystems to represent the logical functions of each of the enterprise systems. This business model contains both the details of the business logic, as well as the mapping of the logic into the major subsystems. The business model forms the basis for managing all changes to the current systems. And the next step is System Integration using Conceptual Model of Platform Specific Models (PSM's), for each of individual systems to form enterprise system [12]. These models were each derived from one or more subsystems in the business model. System construction consists of customizing each of the enterprise systems, and creating the business logic. Business logic that spanned systems is constructed using components technology and deployed in the application server also called web service brokers, please refer figure2.

\section{CONCEPTUAL MODEL OF PROCESS AND TRANSACTION LAYER}

This framework contains an underlying java Foundation made of fundamental data structure implementations and utilities used throughout the rest of Enterprise processes. Examples include arrays, dictionaries and formatting classes. These processes provide RDBMS independence for services persistence, provides object persistence transaction management, and provides services useful for web based presentation and deployment. It also provides an environment to use and create reusable components, it facilitates the use of true business objects in services oriented framework and handles storing and restoring objects to a data store and usually in a relational database. Since the business processes and objects created don't care about the underlying database or 

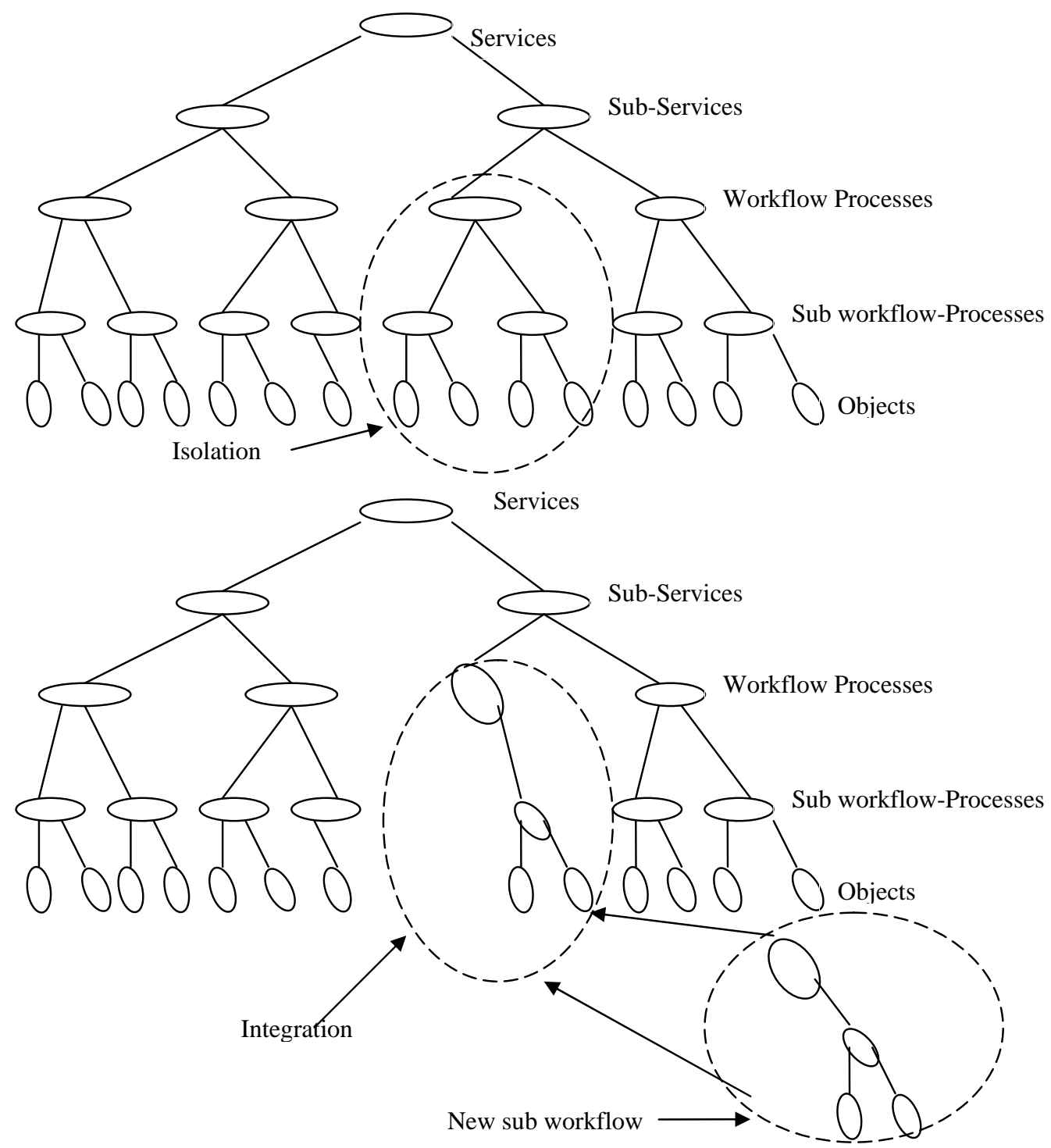

Figure 5. Isolation replacement and integration of new workflow

how their values are presented in user interfaces, they may be re-used over and over in any number of different web applications and can be maintained by developers. Web services framework also provides a persistence layer to maintain information at all time. As a single process can produce a huge workflow map, the subworkflow layers allow the workflow to be broken down into more manageable sections. This also allows modularisation of commonly used functions for example bulk notification activities rather than having them repeated throughout the main map or even several workflows maps or systems. This also makes them easier to manage and maintain. Sub workflow layer can be very useful to split your main process into its constituent elements - in a large process there is likely to produce a quicker initiation and processing. However, in some cases, the overhead of moving from a 'parent' workflow into a 'child' sub workflow can be a lot higher than the performance benefit of doing so, hence we need to plan the workflow carefully.

This type of architecture will help in bringing about main areas of changes like: 


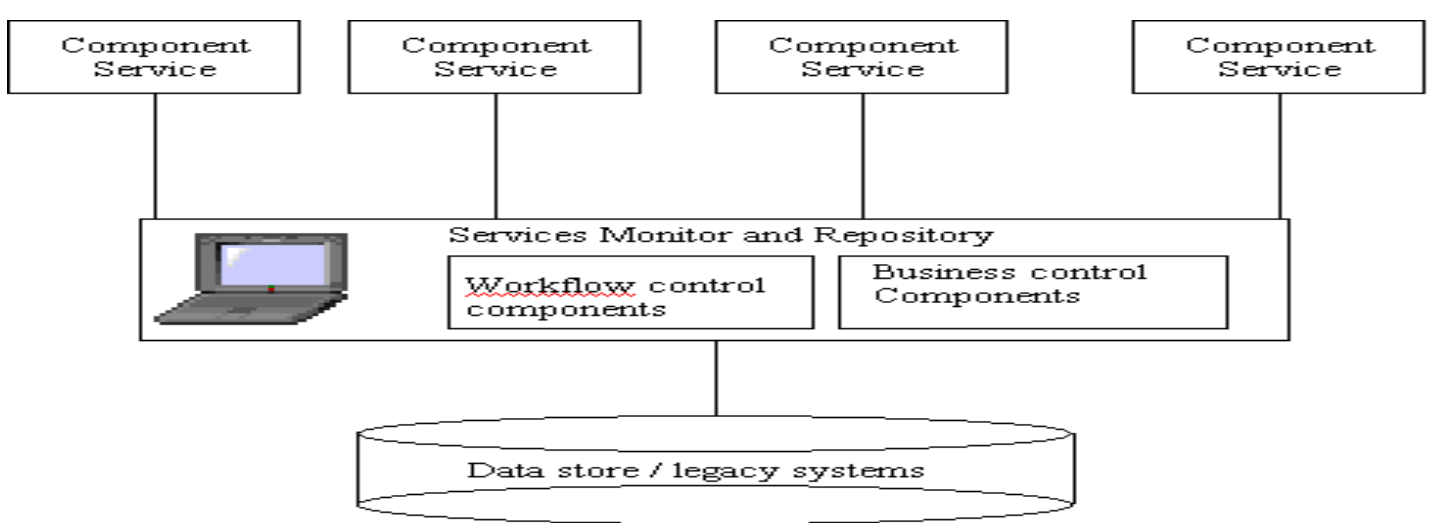

Figure 6. Implementation Framework

Services Layer changes like criteria determining field colouration or visibility or edibility of a given field has changed, or a popup box is now required if some criteria is met.

- $\quad$ Data Changes / Mass Updates like Value Added Tax calculations need to be redone if VAT rate changes, customer name changes

- Business Logic changes like Logistic criteria changes, routing requirements change.

- $\quad$ Patches / Bug Fixes that need to be applied to many active workflows

First case could be handled simply by adding one or more JavaScript functions and some CSS directly onto the affected forms. However, this may result in largescale repetition of code throughout the workflow system which would be difficult to manage and any changes would mean loading in new versions of the affected views. One approach is to suspend, correct and restart each workflow in sequence, although for large numbers of workflows this would be very time consuming [18].

\section{FRAMEWORKS FOR SERVICES COMPONENTS IMPLEMENTATION \\ AND}

We propose use of a modular approach to software development for implementation of this model, current advancement in technology has resulted in better quality, reusability, productivity, and cost effectiveness. Changes to the system composition and configuration are limited once the system has been compiled. In order to solve the complexity and Although transactional integrity mechanisms protect the database from corruption through multiple flexibility issues of large-scale software, We encapsulate business logic in a workflow, and use component based middle layer called Services Monitor and Repository which acts as a centralized server that contains all diagrams, reports, forms, data structure, data definitions, process flows, logic, and definitions of organizational and system components; it provides a set of mechanisms and structures to achieve seamless data-to-tool and data-to-data integration, this middle ware provides the link between Component Services and data store. This services monitor and repository layer follows strict object oriented principles, it contains two major parts, workflow control components and Business control components which contains all the objects that execute the complex business rules.

In data store large complex workflow processes are broken down into smaller workflows and sub workflow layers to be able to better manage and maintain each section. Some process activities may be repeated throughout the main map or even several workflows maps or systems. This allows modularization of commonly used functions and help in easy management by Services Monitor described in detail below. Data store shown in Figure 6 aims to eliminate latency by allowing multiple applications to access a single physical data store directly. This architecture is suitable when applications and databases are located in the same data centre; this approach is more intrusive because we usually have to modify some applications to use a common schema. Reading data directly from a database is generally harmless, but writing data directly into an application's database risks corrupting the application's internal state.

concurrent updates, they cannot protect the database from the insertion of bad data. In most cases, only a 
subset of data-related constraints is implemented in the database itself. [20]. To avoid this we include Services Monitor which is visual tool mapping software which is part of the services monitor and repository layer, we use component technology for data management in order to extract the underlying schema in the datastore which is also in the form of components. Services monitor allows us to identify the workflow processes and sub workflow processes and objects stored in the data source, which need to be isolated and a new sub workflow which has to be integrated, it also helps to create, edit, or delete existing data store objects dynamically when connected to the datastore. We can interact with the server data store using datastore diagrams incorporated in the service monitor. Datastore diagrams graphically represent the tables as of a normal database. These tables display the columns they contain, the relationships between the tables, and indexes and constraints attached to the tables. We can use data store diagrams to: View the tables in your database and their relationships. Perform complex operations to alter the physical structure of the database.

We can make changes freely in the datastore diagram without affecting the underlying datastore. When we modify a datastore object through a datastore diagram, the modifications made are not saved in the datastore until we save the table or the datastore diagram, Visualize the structure of your database tables and their relationships. Provide different visualizations of complex databases. Experiment with database changes without modifying the underlying database. Create new tables, indexes, relationships, and other constraints. Alter the structure of your database. Thus, we can experiment with "what if" and various workflow scenarios and also check if these changes made to the workflow can be integrated to the existing workflow, using a datastore design without having to permanently affect its existing design or data. During editing, we can experiment with different object definitions to see if proposed modification will affect the datastore. When we complete these modifications, we can either save our diagram/design or update the database to match the diagram, or we can discard it leaving the underlying database unchanged.

For example [please refer to figure 7], you can create/view a database diagram for customer services department that shows only tables that hold local delivery of goods information. We can view workflow part of the process that shows only those tables that are used in this specific workflow module, here we can make change to Devanning process to replace Warehouse code with Warehouse type code and Delivery docket with Delivery time. We can change the size, shape, and position of objects in the diagram without affecting their definitions in the database. When we save the datastore diagram, the layout of the diagram is preserved as well as any changes made to the object definitions in the diagram are also saved.

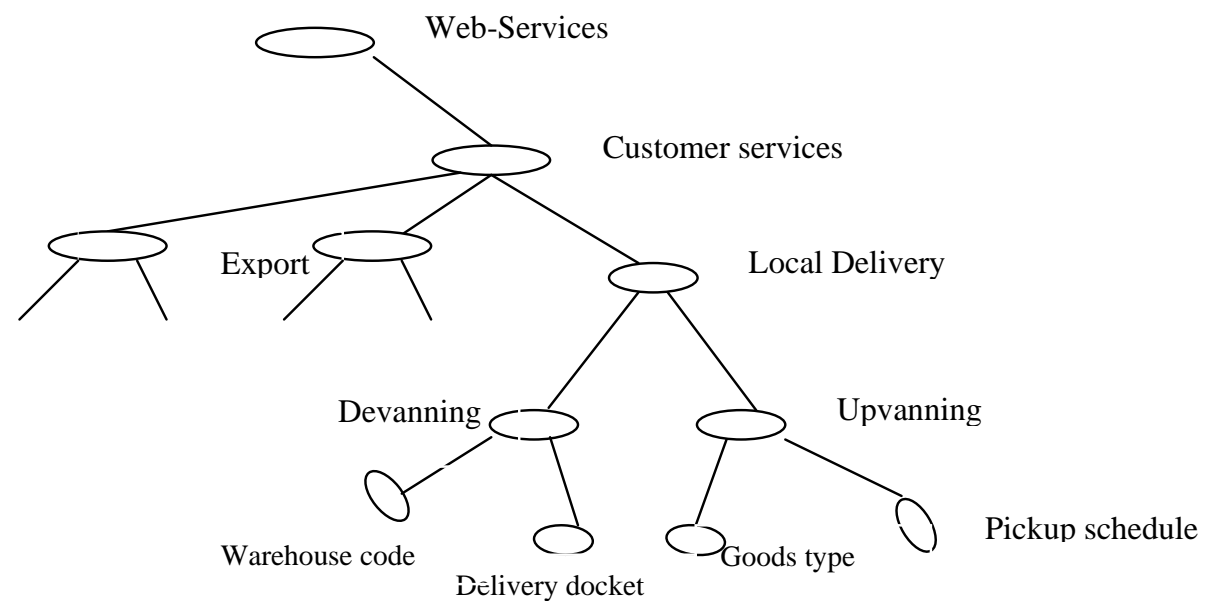

Figure 7. Example of local delivery process 


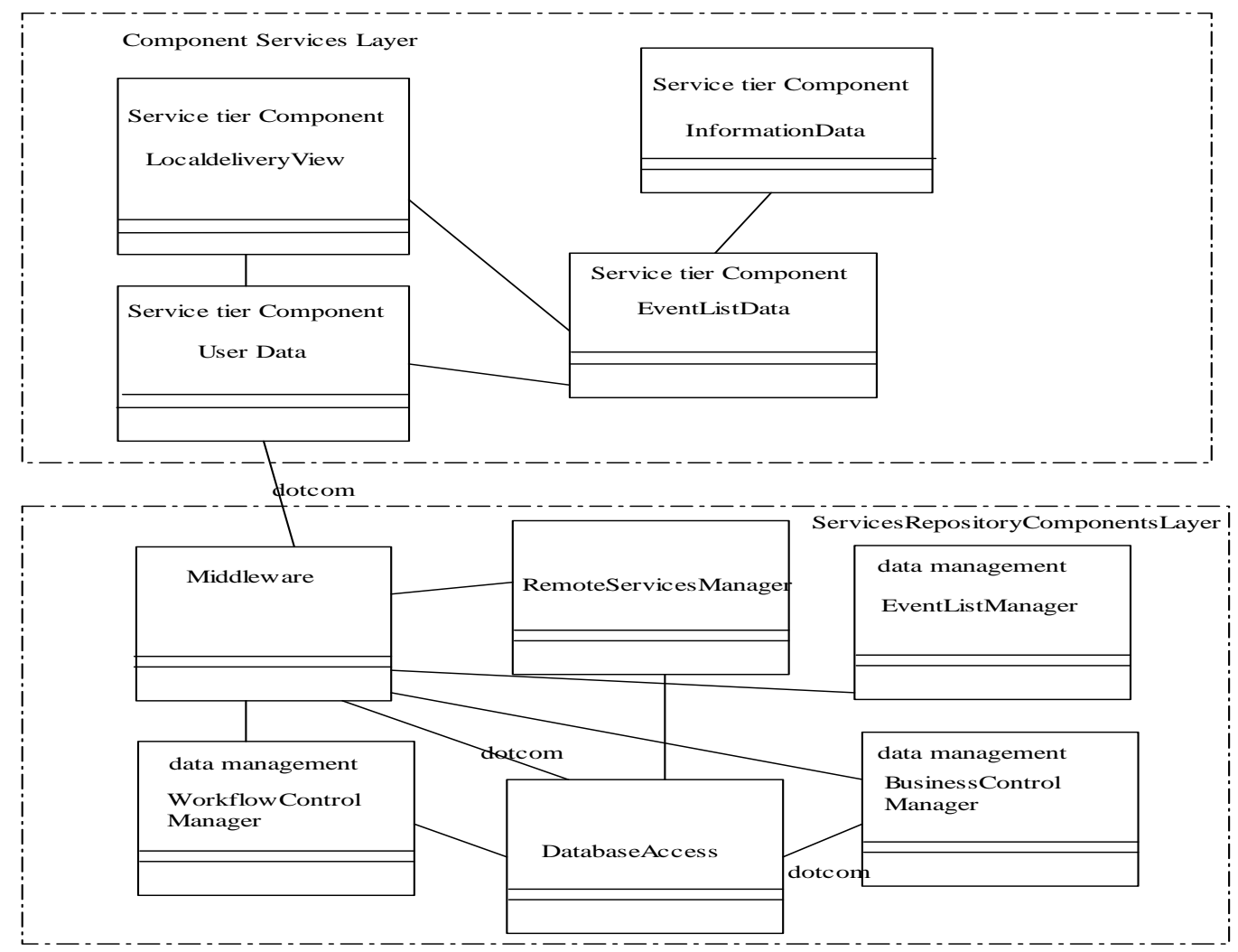

Figure 8 : Example of component design for our services

So as to keep the whole consortium process running we propose exclusive locking mechanism; the locking level Performance and concurrency can also be affected by the locking level used, Exclusive locks are exclusive to the user till the changes are made without having to dissturb the overall workflow. Exclusive lock on a record means that part of the process is denyed access, there by that part of the workflow is isolated so that the required changes can be made only to that part of the process, one may choose some objects or even all of the workflow or sub workflow tasks to be associated with implicit invocation. determines the size of the process that is locked.

[21]. This framework is applicable to a diverse range of software Performance and concurrency can also be affected by the locking level used, Exclusive locks are exclusive to the user till the changes are made without having to dissturb the overall workflow. Exclusive lock on a record means that part of the process is denyed access, there by that part of the workflow is isolated so that the required changes can be made only to that part of the process, one may choose some objects or even all of the workflow or sub workflow tasks to be associated with implicit invocation. When editing of table in a datastore diagram has been done, an asterisk (*) Example Delivery time $^{*}$, appears after the table name in the title bar to indicate that the table contains changes to the workflow that have not yet been saved in the database. This indicator appears as a result of a change made to the workflow objects in the datastore, represented as a column or index, in the table of the diagram/ design. When we add a modified table to another open diagram, the table appears there with its unsaved changes and an asterisk in its title bar. When you save the table or the diagram, the asterisk disappears. This reconfigurable plug and play object component-based framework [please refer to figure 8] is used to specify collaborative software construction, customization, integration and evolution through the reuse of context-independent objects where composite architectures are hierarchically constructed from layered groups of collaborating component plug-ins development environments. 
For user interface web services layer browsers interact with HTTP servers in their normal way taking advantage of any technologies that enhance this browser-to-web server link. For example secure socket layer communication protocols in Netscape and Microsoft browser/server products browsers communicate with HTTP servers, which communicate with the Application Server. The Business web services layer generates web application at run time, Services communication layer provides application's user interface, state management and provides an environment to use and create reusable components [7]. business logic is separated using two tier approach, web services are generated at runtime from metadata in the services repository component layer, this middleware data describes the webservices and its interaction with the underlying business logic components [22]. This procedure helps us to have multiple user services configuration based on shared business and workflow logic components.

\section{CONCLUSION:}

In this paper, we have discussed service oriented architecture for dynamic workflow systems. We have also discussed issues and frameworks, service oriented enterprises systems and have come up with approach for dynamic adaptation of the changes to the existing workflow. We propose implementation of such systems by the process of isolation, integration and synchronization, our future research will be to implement this plug and play software development methodology and come up with a working prototype of our system.

\section{REFERENCE:}

[1] Marshak, R.T 1994.: "Falling in Love with Distinctions", In "New Tools for New Times: The Workflow Paradigm", Future Strategies Inc.,

[2]Miers, D 1996: "The Workware Evaluation Framework", Enix Limited,

[3]Chang, E 2000: Requirement Specification of Logistic Manager for Software Engineering Project, Department of Computer Science and Software Engineering, The University of Newcastle,.

[4]Haake, J.M., Wang, W. 2002: "Flexible Support for Business Processes: Extending Cooperative Hypermedia with Process Support”.
[5]Denning, P.J.,1994 “The fifteen level”, In Proceedings of ACM SIGMETRIC Conference on Measurement \& Modeling of Computer Systems.

[6]Sheth A 1996... "State-of-the-art and future directions", In Proceedings of the NSF Workshop on Workflow and Process Automation in Information Systems.

[7] David Neumann, 2004. An Introduction to WebObjects. Retrieved:July30,from

http://.mactech.com/articles/mactech/Vol.13/13.05/WebObje ctsOverview.

[8] Pudhota L, Chang E. et al 2003,. International Journal, Computer Science, System and Engineering, "Extension of Activity Diagrams for Flexible Business Workflow Modeling "volume 18 no3 UK.

[9] Pudhota L, Chang E 2004 "collaborative workflow management for logistics consortium” ICEIS Porto, Portugal.

[10] Pudhota L, Chang E 2004 "Modelling the Dynamic Relationships between Workflow Components" ICEISl Porto, Portugal.

[11] Pudhota L, Chang E, Venable J 2004 "E- Business technology adaptation through workflow mining” MSV Las Vegas, Nevada, USA.

[12] http://www.omg.org/mda/mda_files/UNextMDA4.pdf [13] Ulieru. M, Robert W. Brennan Scott S. 2000 "The holonic enterprise: a model for Internet-enabled global manufacturing supply chain and workflow management", Canada.

[14] Ulieru. M, Stefanoiu. D, et al2000.. "Holonic metamorphic architecture for manufacturing” University of Calgary, Calgary, Canada

[15] Brandenburger, A. M. and Nalebuff, B. J., 1996, Cooperation, Doubleday NY.Brennan, R. 2000, "Performance comparison and analysis of reactive and planning-control architectures for manufacturing", Robotics and Computer Manufacturing 16(2-3), pp. 191-200.

[16] Christensen, J.H, 1994 "Holonic Manufacturing Systems: Initial Architecture Standards Directions", Proceedings of the First European conferenceManufacturing systems, European HMS Consortium, Hanover, Germany.

[17] http://www.journee.com/n_hl_020703b.html

[18] Anastassia.A, Yannis E. et al 1998 "Scientific Workflow Management by Database Management" book title = "Statistical and Scientific Database Management" pages = "190-199",

[19] Clark, M., Fletcher, P., et al 2002. Web Services

Business Strategies and Architectures. Expert Press.

[20] Talevski, A., Chang, E., Dillon, T., 2003 "Overview of a

Plug and Play Component-Based Framework”, World

Multiconference on Systemics, Cybernetics and Informatics, Orlando, Florida, USA.

[21] Maloney, J., “COM Reality Tour”, Microsoft

Cooperation. On-line at: http://www.microsoft.com/com/ August 1997

[22]T. Andrews, F. Curbera etc.: Business Process Execution Language for Web Services Version 1.1. http://www-

106.ibm.com/developerworks/webservices/library/ws-bpel/, May 2003. 